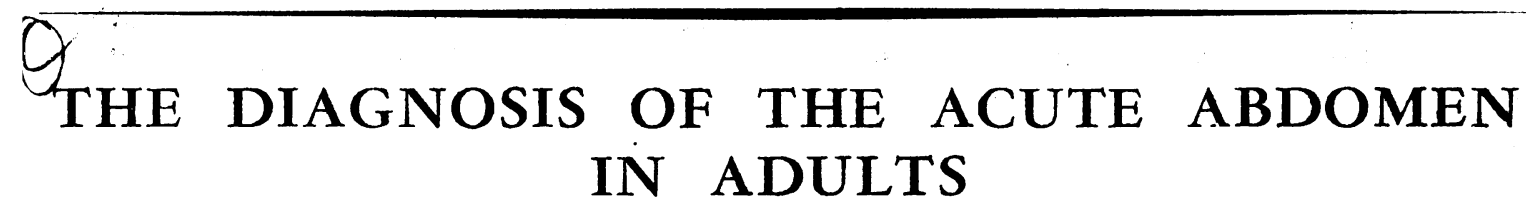

\author{
By ANDRew Monro, M.D., F.R.C.S. \\ Lecturer in Surgery, Postgraduate Medical School of London; Surgeon, Southend General and St. Yohn's Hospitals
}

\begin{abstract}
'Experience informs us only of what has been, never of what must be.'-Reid.

The diagnosis of the acute abdomen offers the
\end{abstract} most immediate personal challenge to all concerned in its management. In no branch of the art of medicine are the penalties of error greater, not only in mortality but in duration of illness and in future ill health for the patient. Moreover the decisions upon which so much depends must be made at the bedside, without delay, often in the small hours of the morning when few of us are at our best.

On the patient's side there has been a change of attitude. Gone are the days of overflowing gratitude for miraculous cures. He now expects to be cured quickly and smoothly and is apt to be highly critical of any ineptness on the part of his medical attendants. Recent litigation has drawn attention to the doctor's responsibilities in these matters.

The subject of the acute abdomen, as we know it, was born and has reached its present stature within living memory. The first successful removal of a ruptured ectopic gestation (Lawson Tait, 1883), of an acute appendix (Hall, 1886) and closure of a perforation (Kriege, 1892 ) were made possible by the coeliotomists who, greatly daring, first ventured to attack the abdominal cavity in open defiance of Lord Lister's contemporary teaching. Gunshot wounds of the abdomen were not treated routinely by surgical exploration until the Russo-Japanese War of 1904 (in a Red Cross train). Two world wars and the advent of antibiotics have vastly extended the scope of the subject and made more than ever important an early and exact diagnosis. An early exact diagnosis and the courage of one's convictions to act upon it are the foundations of good treatment in these conditions.

The diagnosis of the acute abdomen depends firstly on the collection of evidence from four sources:

I. The history.
2. Physical examination.

3. Certain ward procedures.

4. The special departments.

\section{The History}

In the patient's history, firstly, previous illnesses or operations may have a bearing in the present emergency, as may previous attacks. Then the mode of onset is often of cardinal importance. This is particularly true in the so-called ' missed' appendix, seen perhaps on the fifth day of the disease. Careful questioning as to the exact modg of onset in such a case may be a most importan pointer to the correct diagnosis. In the case of abdominal injuries the story of the exact mode o injury obtained from the patient or from witnesses may be of similar value.

\section{Physical Examination}

The more complete the doctor's examination in the patient's home the more valuable will his evidence be. By the time the patient reaches hospital the physical findings may have altered and a knowledge of their development is important. None the less it is by no means easy nor, indeed, always possible for a busy practitioner to carry out a full examination in a cold back bedroom in a bad light. If he decides from abdominal examination alone that hospital admission is essential and proceeds to effect this forthwith, I believe that he has exercised reasonable skill and care. The responsibility then passes to the receiving hospital. None the less a careful letter mentioning previous history, mode of onset, physical signs and including the doctor's provisional diagnosis may be as valuable as any evidence gathered subsequently.

Physical examination must be complete, from the crown of the head (at least from the expression of the forehead and the reactions of the pupils) to the soles of the feet (for the plantar reflexes). The presence of Argyll-Robertson pupils, of glands in the supraclavicular fossa, of a tumour in the 
breast, of a pleural rub, of arteriosclerosis or of a swelling in a testis may be the key to the diagnosis.

In the examination of the abdomen exposure from the nipples to the knees in a good light is sound practice. A few moments spent in inspection from the bottom of the bed may show not only fullness in the flanks, types of peristalsis and so on, but also movement of the abdomen generally and on respiration, and whether voluntary movement is painful.

Palpation is directed first to the external hernial orifices. Most of us must admit to having overlooked a strangulated femoral hernia in an obese patient. Such a swelling is not always tender. The writer has seen such a hernia containing gangrenous bowel in a woman of 72 who was not even aware of the presence in her groin of a non-tender swelling the size of a plum. Another pitfall is the presence or absence of an impulse on coughing. Every swelling related to the anterior abdominal wall gives a heave on coughing; the point at issue is the presence or absence of an expansile impulse, that is of an actual increase in volume of the swelling on coughing. The absence of an expansile impulse on coughing in a hernia is the essential sign of strangulation. It is superfluous to add that in the case of a femoral hernia without such an impulse, any attempt at taxis is absolutely contra-indicated. If taxis is applied in such cases a gangrengus constriction ring may be ruptured with undoubtedly fatal result. On the question, therefore, of whether a strangulated femoral hernia is reducible or not, ignorance is both bliss and wisdom.

In palpation of the abdomen a direct approach to the suspected area with a firm hand is most unwise. The value of the examination may be thereby entirely lost, together with the patient's confidence. The prudent clinician starts his examination at the point of the abdomen most remote from the suspected area with the lightest possible hand. Is the slightest movement of the peritoneal surface painful? Is such tenderness localized? Gradually he moves round the abdomen to reach the suspected area last. In the absence of marked tenderness, deeper palpation is then permissible with a view to feeling a mass of viscus. Repeatedly an inflamed or even gangrenous appendix is removed from an abdomen whose only abnormal physical sign was slight tenderness in the right iliac fossa. This, combined with a suggestive history, is not infrequently the only evidence of acute appendicitis in the early stage in which we wish to remove it. Full-blown tenderness and rigidity may appear only subsequently and may be late signs of the disease. It is the gentle hand which learns most.

Percussion of the abdomen, carried out with the shoulders propped up, may reveal diminution or absence of liver dullness after perforation of a hollow viscus, or may show shifting dullness, indicating free fluid. Its value as a physical sign, however, is not to be compared with that of auscultation. In any question of peritonitis, the presence, absence or change of bowel sounds heard with the stethoscope is of prime importance. In suspected perforations and in abdominal injuries, particularly, the stethoscope gives most valuable information, the persisting absence of bowel sounds indicating a general peritonitis. The stethoscope is by no means the prerogative of the physician.

Last, but by no means least, is pelvic examination. Lesions in the pelvis are within reach of the examining finger. To learn most it must be gentle and unhurried. In the cavity of the rectum may be blood or the head of an intussusception. In the wall of the rectum may be a carcinoma or the lower extremity of an area of diverticulitis. Outside the rectum may be a tender bulging of the rectovesical pouch in an early general peritonitis, or of the pouch of Douglas in a ruptured ectopic gestation. Again it is the gentle finger which learns most. Is the slightest movement of the terminal segment of the finger painful, causing only the gentlest movement of the peritoneal surfaces? More remotely, primary lesions of the colon or secondaries on the floor of the pelvis may be felt More remotely still, an obturator hernia, giving? characteristic pain down the inner side of the thigh to the knee, aggravated by coughing and accompanied by tenderness deeply below the inner end of Poupart's ligament, has been diagnosed correctly by feeling, from the vagina, the mass of the bowel running to the obturator foramen.

\section{Certain Ward Procedures}

There is no exception to the golden rule that a catheter should be passed in all cases of abdominal injury. Its omission may be sincerely regretted.

Aspiration of the stomach with a Ryle's tube may give evidence of importance. Failure to be able to pass the tube has been diagnostic of a torsion of the stomach.

A single enema may give evidence as to the position of an obstruction in the colon. If half a pint of fluid is the maximum which can be retained, the lesion may well be in the sigmoid colon; if a pint and a half or two pints are retained, the lower colon is exonerated. A second enema repeated an hour later (' the two enema test ') may corroborate a diagnosis of intestinal obstruction, the salient point being the presence or absence of flatus when the second enema is returned. It is again superfluous to mention that no enema is ever ordered in these conditions before a rectal examination has been done-for obvious reasons. 


\section{Special Departments}

$X$-rays. The special departments give valuable and sometimes conclusive evidence. None the less that evidence must be interpreted in the light of the other findings in the case. For instance, the presence of free gas under the diaphragm, in the absence of recent laparotomy, is proof of perforation of a hollow viscus. On the other hand, the presence of fractured ribs on the left side posteriorly does not by any means rule out the possibility of a ruptured spleen, an association which has caused the gravest difficulties.

In non-traumatic conditions $\mathrm{X}$-rays may be helpful in showing the presence of gas under the diaphragm, in showing the type or site of an intestinal obstruction, the position of the caecum, of a volvulus, of a gall stone in gall stone obstruction and in other conditions. In traumatic conditions X-rays are, of course, useful in showing skeletal fractures as well as in showing the presence of free gas or of retained foreign bodies.

In many cases their role in showing the state of the bases of the lungs and of the costophrenic angles is most important in ruling out disease above the diaphragm, whilst screening of the diaphragmatic movements is of cardinal importance in the diagnosis of subphrenic infection.

The pathology and biochemistry laboratories are invaluable in the confirmation of a diagnosis of pancreatitis. In other cases they are helpful in the examination of blood, of urine and of stools. The white cell count is held to be of considerable importance in these conditions in the United States. Certainly it may be of help in the diagnosis of a difficult case. So frequently, however, it gives mere corroboration of the thermometer reading that we do not consider it here a routine procedure. If on the other hand the patient is to be kept under observation for any time, variation in the white cell count may be important and under these circumstances repeated counts should certainly be carried out.

The finding of pathogenic amoebae in the stools may give the solution to otherwise insoluble problems.

Electrocardiography in doubtful cases may similarly be invaluable in cases of cardiac infarction.

\section{Process of Diagnosis}

At each stage in the management of the acute abdomen the doctor concerned should sum up his findings by making a diagnosis. The general practitioner, having taken the history and made as thorough an examination as circumstances will allow, should write his letter mentioning the salient points of the history, mode of onset and physical findings at the time of examination, not omitting such basic information as the temperature and pulse rate when first seen. His opinion as tof diagnosis rounds off his letter, acts as a stimulusz both to himself and to those subsequently con $\frac{\bar{D}}{2}$ cerned and invites the courtesy of a reply when the. facts are known. Such a letter can be of the greatest importance:

A railway shunter was crushed between tw trucks in the shunting yard. The doctor who carried out first aid noticed tenderness and rigidity in the left upper abdomen and wrote "probable ruptured spleen.' On arrival at hospital 20 mines later the patient was unconscious from massiveintraperitoneal haemorrhage from an avulsed splenic pedicle and was saved only by immediate transfusions and surgery.

The doctor's observation directed the searcio and saved precious minutes. Again an observation. in a doctor's note that five years previously an obese and incoherent woman had suffered fromiw gall stone colic suggested a diagnosis of gall stoner obstruction which was duly relieved surgically. N

In the Casualty Department again, the admitting officer after due examination should always make a diagnosis, tentative obviously, but again important.When he, later in his career, is faced with the ultimate decision in such cases, he is meeting problems he has faced before. The house surgeono proceeds similarly.

It is in the summing up of evidence, in the making of a final pre-operative diagnosis, thap experience plays its part. Its first lesson is that in these cases no diagnosis is ever cast iron; it is an opinion based on probabilities. Even where diagnosis appears most certain, as in some cases of acute appendicitis, it must be remembered thae conditions in the anterior abdominal wall, in the abdominal cavity and behind the cavity may proz duce an identical picture. A haematoma of the right rectus muscle occurring after an attack of bronchitis, a leaking aneurysm of the common iliag. artery or haemorrhage into para-aortic glands. secondary to carcinoma of the testis can all veris closely mimic such a case. Obviously failure tक् make the patient contract his recti, to observe the absence of a femoral pulse or to palpate a tumour? in the testis are serious omissions.

Again experience helps us in weighing the evidence, to give weight where weight is due. In the history the exact mode of onset is probably the most important point, followed by the changesu since. In the physical examination the degree of tenderness, particularly to light touch, changes in bowel sounds and pelvic examination, particularlye with regard to the slightest bulging of the rectoen vesical pouch, or to pain caused by the slightest movement of the distal segment of the finger are the most important points.

Experience is of value also in the interpretation? 


\begin{tabular}{|c|c|c|c|c|c|}
\hline \multicolumn{5}{|c|}{ ACUTE ABDOMEN } & \multirow[b]{2}{*}{ Diagnosis } \\
\hline Peritonitis & $\begin{array}{c}1 \\
\text { Intestinal } \\
\text { Obstruction }\end{array}$ & Torsions & $\begin{array}{c}1 \\
\text { Internal } \\
\text { Haemorrhage }\end{array}$ & Differential & \\
\hline $\begin{array}{l}\text { Tenderness and } \\
\text { rigidity }\end{array}$ & $\begin{array}{l}\text { Persistent } \\
\text { vomiting }\end{array}$ & A tender mass & General signs & Colics & Retroperitoneum \\
\hline & $\begin{array}{l}\text { Colicky pain } \\
\text { Abdominal } \\
\text { distension }\end{array}$ & & Local signs & $\begin{array}{l}\text { Medical con- } \\
\text { ditions (hepatitis } \\
\text { enteritis) }\end{array}$ & Abdominal wall \\
\hline & Constipation & & & $\begin{array}{l}\text { Chest } \\
\text { conditions }\end{array}$ & $\begin{array}{c}\text { Neurological } \\
\text { conditions } \\
\text { Eye conditions }\end{array}$ \\
\hline
\end{tabular}

of the findings of the special departments, particularly in the interpretation of negative findings. Can a patient have an acute appendicitis with a white cell count of only 6,000 or a perforation without evidence of gas under the diaphragm? (in both cases, yes).

With the evidence so obtained a large percentage of abdominal emergencies fit into certain well recognized groups. A full classification is complicated and easily forgotten, particularly at dead of night when most needed. The above is a simple classification which is easily remembered.

Some three-quarters of all the emergencies with which we have to deal fit into one or other of these groups with a fairly firm diagnosis. In the remaining quarter the diagnosis is still in doubt. For these there are three possible lines of procedureexclude, expect or explore. In all cases careful examination is carried out to exclude extraabdominal causes: above the diaphragm (heart, lungs, pleura, diaphragmatic hernia), behind the peritoneum (renal causes, glands, leaking aneurysm, etc.), in the anterior abdominal wall (haematoma of the rectus), neurological causes (pre-eruption zoster, nerve root irritation, tabes dorsalis) and glaucoma (the writer has yet to see a case so confused). Medical conditions within the abdomen must also be considered, particularly colics, infective hepatitis and enteritis of whatever kind.

Such causes having been as far as possible excluded, there are certain cases which must be opened forthwith. If haemorrhage, strangulation or progressive inflammatory lesion in the abdominal cavity is present, reflected in the patient's serious or worsening general condition or in extension of local physical signs, intervention is indicated. Intervention is also wise where the appendix may be to blame.

In the absence of these indications, expectant measures may be adopted in surroundings where (a) the patient can be kept under constant observation with an hourly or half-hourly pulse chart, and (b) there are full facilities for immediate surgical intervention should this prove necessary. Such facilities are not present in the patient's home and expectant treatment should therefore never be carried out there. If conservative measures are adopted the greatest care must be taken not to allow resuscitation to foster a sense of false security. Delay may settle the diagnosis; it must not be allowed to settle the prognosis.

A note on the changed outlook to abdominal emergencies in geriatrics is timely. Until recently, in those of advancing years, the approach has been to operate only if one has been forced to do so. Experience has shown, however, how serious are the complications of keeping old people in bed, chest complications, retention of urine, thromboses and embolisms being all too common. Moreover the penalty for missing an early appendix in old age is usually extreme. On the other hand, early diagnosis and operation combined with early mobilization has given encouraging results. In advancing years therefore, early diagnosis remains just as important and should be pursued to the full, with planning of the incision if operation is undertaken, to allow early mobilization.

In cholecystitis, diverticulitis, sub-acute obstructịons, mesenteric adenitis, Crohn's disease and other conditions, conservative measures may be good treatment. To miss an early appendicitis is very bad treatment. On the whole, when in doubt it is better to be safe than sorry; it is better to look and see rather than to wait and see.

\section{Summary}

I. Diagnosis of the acute abdomen must be early and exact.

2. General practitioner, casualty officer and house surgeon should each, in turn, make and record his diagnosis.

3. A careful letter from the general practitioner may be as important as any subsequent evidence.

4. In doubt exclude, perhaps expect, probably explore. 
5. The passage of time may settle the diagnosis; beware lest it also settle the prognosis.

6 . In the absence of haemorrhage, two hours or more are well spent in pre-operative preparation. This is resuscitation, not procrastination.

7. Exploration does not necessarily involve a long paramedian incision. A gridiron incision takes little time; may be of vast advantage to the patient, but requires the strength of the surgeon's $\frac{3}{\infty}$ convictions.

\section{BIBLIOGRAPHY}

HALL, R.' J. (1886), N.Y. med. F., 43, 662. KRIEGE (1892), Berlin klin. Wchnschr., 29, 1244. LAWSON TAIT (1884), Brit. med. Y., I, 1250.

\title{
S THE PLACE OF RADIOLOGY IN THE MANAGEMENT OF THE ACUTE ABDOMEN
}

\author{
By J. W. Prerce, M.D., M.R.C.P., D.M.R.D. \\ St. Thomas's Hospital, London
}

\section{Introduction}

For reasons of time, place-or preferencemost acute abdominal emergencies in adults are successfully treated without $\mathrm{X}$-ray examination. Many surgeons, however, prefer to have an X-ray of the abdomen in all save the most obvious cases, but the practice varies in different hospitals, and appears to be governed less by logic than tradition. In infants and children, the possibility of underlying congenital malformations has always to be considered and in this field the importance of radiology is generally accepted.

There is no point in submitting a patient to the discomforts, delays - and possible dangers - of an $\mathrm{X}$-ray examination unless the result may either demonstrate that surgical intervention is unnecessary or enable the surgeon to modify his approach. Many acute cases present a clinical picture clear enough to warrant immediate operation, while the condition of others may demand a laparotomy no matter what an $\mathrm{X}$-ray could show; radiology is therefore often superfluous. But in patientsparticularly those with the not-so-acute abdomen -when the diagnosis is not reasonably certain, and the course of treatment as yet undecided, radiology can be very helpful, and should be undertaken whenever possible. It is also of great value in cases where observation is thought preferable to immediate operation, or when it is important to localize a lesion as accurately as possible. There are three main ways in which an X-ray examination can help.

(a) By demonstrating free air in the peritoneal cavity it may confirm a diagnosis of gastro-intestinal perforation.

(b) By showing loops of distended bowel it may indicate the presence of intestinal obstruction or ileus and provide some idea of the level of the $\vec{G}$ lesion.

(c) By showing calculi, areas of calcification, $\vec{z}$ skeletal or soft tissue abnormalities; radiology may suggest that the lesion lies elsewhere than in the alimentary tract.

\section{Radiography}

The examination should be carried out quickly, and with as little disturbance to the patient as possible. It is essential to have one film to show fluid levels, and for preference this $\frac{\circ}{\circ}$ should be taken with the patient sitting up. If $\stackrel{\varrho}{\rightarrow}$ this is not possible then it should be taken with aô horizontal ray-across the X-ray table-the patient lying on his left side. Although the examination should be done quickly it must not be hurried, as films of indifferent quality are worse $\frac{0}{3}$ than useless-they are dangerous. It is notalways easy to obtain films of diagnostic quality from a portable set, and whenever possible the films should be taken in the X-ray department. $\frac{\text { S }}{3}$ Only rarely are the patients too ill for this to be done, but in dealing with acute admissions the decision to take an X-ray must be made early, so $\frac{7}{0}$ that it may be done on the way to the ward, and not left until the patient has been wearied by $N$ repeated examinations.

An X-ray of the chest should always be taken, $\stackrel{N}{N}$ preferably with rather more penetration than usual, $\omega$ so that shadows behind the heart can be seen.

\section{Findings}

\section{Perforations}

Free air is usually found immediately under the domes of the diaphragm, above the liver or ${ }^{\circ}$ stomach. Very small amounts can be demon- $\stackrel{\mathbb{Q}}{\overparen{D}}$ strated, but these cases must be examined carefully $\overrightarrow{\mathbb{Q}}$ 